\title{
Security performance analysis of SIMO relay systems over Composite Fading Channels
}

\author{
Jiangfeng Sun ${ }^{1,2^{*}}$, Hongxia $\mathrm{Bie}^{1}$ and Xingwang $\mathrm{Li}^{3}$ \\ ${ }^{1}$ School of Information and Communication Engineering, Beijing University of Posts and Telecommunications \\ Beijing, 100084 - China; \\ [e-mail: sunjiangfeng@bupt.edu.cn, biehx@bupt.edu.cn] \\ ${ }^{2}$ College of Computer Science and Technology, Henan Polytechnic University \\ Jiaozuo, 454000 - China; \\ ${ }^{3}$ School of Physics and Electronic Information Engineering, Henan Polytechnic University \\ Jiaozuo, 454000 - China \\ [e-mail: lixingwangbupt@gmail.com] \\ *Corresponding author: Jiangfeng Sun
}

Received December 25, 2019; revised February 26, 2020; accepted March 25, 2020; published June 30, 2020

\begin{abstract}
In this paper, we analyze the secrecy performance of single-input multiple-output (SIMO) relay systems over $\kappa-\mu$ shadowed fading channels. Based on considering relay model employing decode-and-forward (DF) protocol, two security evaluation metrics, namely, secure outage probability (SOP) and probability of strictly positive secrecy capacity (SPSC) are studied, for which closed-form analytical expressions are derived. In addition, Monte Carlo results prove the validity of the theoretical derivation. The simulation results confirm that the factors that enhance the security include large ratio of $\left(\mu_{D}, \mu_{E}\right),\left(m_{D}, m_{E}\right),\left(L_{D}, L_{E}\right)$ and small ratio of $\left(k_{D}, k_{E}\right)$ under the high signal-to-noise ratio regime.
\end{abstract}

Keywords: Cooperative relay, composite fading, performance analysis, physical layer security, decode-and-forward

\footnotetext{
This work was supported in part by the National Natural Science Foundation of China under Grant 41174158 and in part by the Ministry of Land and Resources P.R.C Special Project in the Public Interest under Grant 201311195-04, in part by the Henan Scientific and Technological Research Project under Grant 182102210307, the Fundamental Research Funds for the Universities of Henan Province under Grant NSFRF180309, the Outstanding Youth Science Foundation of Henan Polytechnic University under Grant J2019-4, the National Natural Science Foundation of China under Grant 61601414.
} 


\section{Introduction}

Different from traditional approaches of solving the security problem of wireless communication, physical layer security (PLS) focuses on utilizing the characteristics of the transmission channels, rather than relying on the high complexity encryption/decryption system [1]. In recent years, the research on PLS has made a gratifying progress [2-8]. Wang et al. in [2] developed a integration framework to improve the PLS performance of internet of things (IOT) networks. Moreover, the IOT networks requires that heterogeneous terminals can communicate directly, so cross-technology communications (CTC) technology emerges as the times require [3]. The authors of [4] studied the security performance of CTC communication by using the inherent characteristics of physical layer. In [5], the derivations of SOP and PNSC of cognitive radio networks over Nakagami- $m$ fading channels were obtained. Lei et al. provided security performance analysis for different generalized fading channels including generalized Gamma [6] and $\alpha-\mu$ fading [7]. To investigate the security performance of free space optical (FSO) networks, the lower bound of SOP and SPSC over mixed model composed of Rayleigh and generalized- $K$ fading channels were given in [8].

As a promising technology to overcome the adverse effects of signal attenuation in the wireless communication networks (WCNs), relaying schemes that can significantly improve the transmission range and quality have gained great attention in recent literature [9-17]. Chen et al. in [9] studied confidentiality of a cooperative relay network over Rayleigh fading channels by deducing SOP in two scenarios, namely, full duplex and half duplex. In [10], the security analysis of satellite communication systems over Rayleigh fading channels with multiple eavesdroppers was investigated. The authors of [11] studied the security performance of single-antenna relay systems over generalized- $K$ fading channels, where the SOP, probability of strictly positive secrecy capacity (SPSC) and ASC were presented based on amplify-and-forward (AF) protocol. In [12], Peppas et al. considered a composite channel model in which the link from the source to the relay node undergoes $\eta-\mu$ fading and the channel from the relay to the target node is subject to $\kappa-\mu$ fading, In particular, closed-form expressions for the OP and average bit error probability (ABEP) were given for analysis. On the premise of fully considering the unfavorable factors in communication networks, the exact and approximate OP for relaying systems over Weibull distribution were presented in [13]. The authors in [14] proposed a relay system in which the relay node was based on AF with multiple antennas. According to this model, ASC was derived to analyze the security performance. In [15], a decode-and-forward (DF) relay model was provided, and OP and IP were derived over Nakagami- $m$ fading channels. The performance of the relaying non-orthogonal multiple access (NOMA) networks over Rayleigh fading and $\alpha-\mu$ fading were analyzed in [16] and [17], respectively.

It is worth mentioning that the use of multi-antenna is an important measure to improve the channel capacity of WCNs and is applied to practical scenarios such as mobile phone communication [18], radar networks [19], vehicle-to-vehicle systems [20], etc. Recently, many scholars have investigated the enhancement of PLS with the aid of multi-antenna technologies. Zhou et al. in [21] analyzed the security performance for the Wyner's model with multiple antennas over $K$ fading channels, elaborated the derivation of ASC, SOP and SPSC, and verified them by Monte Carlo simulations. The authors of [22] proposed a multi-antenna composite relaying system, where the channel from the signal source to the relay and to the eavesdropping user experiences Nakagami- $m$ fading, while the link from the relay to the 
legitimate receiver undergoes generalized- $K$ fading, Moreover, the closed-form OP and IP were derived for secrecy analysis. The study [23] presented a simultaneous wireless information and power transfer (SWIPT) system over Rayleigh fading channels with multi-antenna receivers, and theoretical SOP and ASC were obtained. The confidentiality of SIMO system over generalized- $K$ fading channels was analyzed in [24]. In [25], the PLS of the smart city model over two Nakagami-m fading was studied, where the transmitter is equipped with multiple antennas, and the influence of the number of antennas on the system security was discussed. By approximating the probability density function (PDF) of $\alpha-\mu$ distribution to bivariate Fox's H-function, the authors of [26] deduced and analyzed the SOP of multi-antenna network under consideration, and presented the influences of shape parameters ( $\alpha$ and $\mu$ ) on the secrecy performance.

In recent years, the related work mainly involves the researches of statistical characteristics, physical layer performance and security performance on the $\kappa-\mu$ shadowed fading channels. The $\kappa-\mu$ shadowed distribution is a composite model, which considers both multipath fading and shadow fading simultaneously, and can simulate the environments of inhomogeneous scattering, for instance, IoT networks [27], double-hop satellite communication [28], and fifth generation (5G) transmission [29]. The author in [30] proposed the $\kappa-\mu$ shadowed model and presented its statistical characteristics including PDF, cumulative distribution function (CDF) and moment generating function (MGF). Aiming to reduce the complexity of integral calculations, a concise formula on bit error rate (BER) was given based on the approximation of $Q$ function [31]. To analyze the performance of mult-hop networks experiencing $\kappa-\mu$ shadowed fading channels, the ABEP based on optimum relay positioning (ORP) and optimum power allocation (OPA) protocols were derived by the MGF in [32]. In [33], the authors studied the performance analysis of the WCNs with double hop AF relay over the $\kappa-\mu$ shadowed fading channels. Srinivasan et al. in [34] studied the secrecy performance of Wyner's eavesdropping networks over the $\kappa-\mu$ shadowed fading channels, and obtained the approximate SOP and SPSC. Relying on the proposed cognitive radio network (CRN) model, the authors of [35] provided the moment matching expression for PDF on $\kappa-\mu$ shadowed distribution, and derived the exact and approximate $\mathrm{OP}$ to analyze the influence of parameters on system performance. To explore the performance of AF relaying systems undergoing $\kappa-\mu$ shadowed fading, the precise and approximate OP and average capacity were provided in [36]. Sun. et al. in [37] studied the secrecy performance of single-input single-output (SISO) networks over the $\kappa-\mu$ shadowed fading channels, and obtained the approximate SOP and SPSC. As a progressive study of [37], the confidentiality for correlated SIMO $\kappa-\mu$ shadowed channel were investigated in [38]. To explore the PLS of AF relaying systems undergoing $\kappa-\mu$ shadowed fading, the precise and approximate OP and average capacity were provided in [39]. The OP of CRNs considering DF multiple relays over $\kappa-\mu$ shadowed fading was obtained by Nakagami- $m$ approximation in [40].

According to the best of the authors' knowledge, there has been no published literatures involving the PLS of relaying $\kappa-\mu$ shadowed systems, let alone the multi-antenna scenarios. Motivated by exploring the PLS performance of communication scenarios that can be simulated with relaying networks over $\kappa-\mu$ shadowed fading channels, in this paper, we first present a realistic relay eavesdropping model with multiple antennas. After that, based on the DF relay protocol, we derive closed-form analytical formulas of two important scerecy performance benchmarks, namely SOP and SPSC. Finally, we provide a comparison between the numerical results of statistical and theoretical simulation, and discuss the factors that affect the confidentiality of the proposed system. 
The subsequent contents are arranged as follows. Section 2 provides a mathematical model of the relay system under consideration and an overview of $\kappa-\mu$ shadowed fading distribution. In section 3, we derive the closed-form representations of SOP regarding the DF relay eavesdropping model. The theoretical SPSC is given in section 4 . The numerical results and some discussions on improving the secrecy performance are presented in section 5. Section 6 concludes the paper and outlines the main findings of this work.

\section{System Model and Channel Characteristics}

\subsection{System Model}

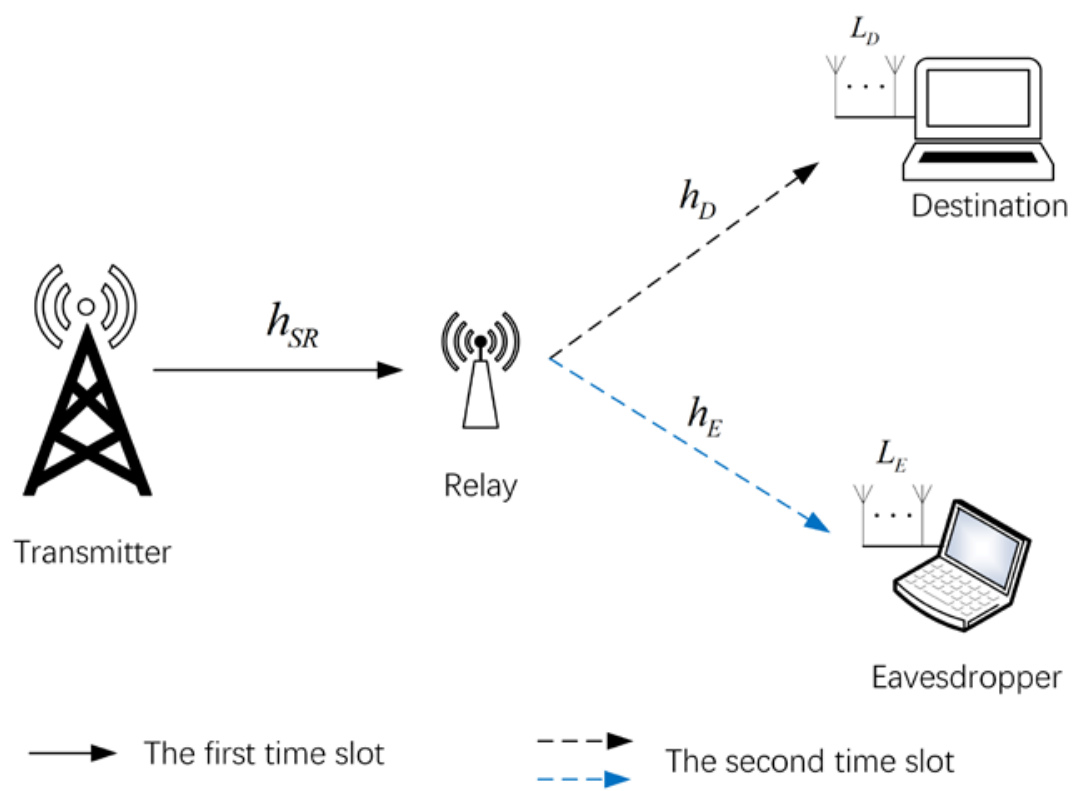

Fig. 1. The system model of the considered scenario

The considered system model with four-nodes is depicted in Fig. 1, where the transmitter $(S)$ is responsible for sending confidential signals, the relay $(R)$ decodes and forwards the signals, the destination $(D)$ represents the expected receiver, while the eavesdropper $(E)$ attempts to intercept confidential information. $D$ and $E$ are equipped with multiple antennas, and the combination method is maximum ratio combining (MRC), while $S$ and $R$ are single antenna nodes, and all links ( $S \rightarrow R, R \rightarrow D, R \rightarrow E$ ) experience independent and identically distributed $\kappa-\mu$ shadowed fading. We assume that the relay is based on DF protocol, and the relay node $(R)$ master the channel state information (CSI) of main link, but has no information about the eavesdropping link. It should be noted that due to severe fading, there are no direct connection on links of $S \rightarrow D, S \rightarrow E$ [41] [42]. the system model can be can be used to evaluate the PLS performance of SIMO WCNs (device-to-device communication, 5G, etc.) with relay nodes.

The communication in the considered relay network consists of signal transmission in two time slots. At the first time slot, a confidential message $x$ is sent by $S$ to $R$, and the message received by $R$ is written as

$$
y_{R}=\sqrt{P_{S}} h_{S R} x+z_{S R}
$$


where $h_{S R}$ is the complex channel gain between $S$ and $R$, and $Z_{S R}$ denotes additive white Gaussian noise (AWGN) with zero mean and variance $\sigma_{S R}^{2}, P_{S}$ is the transmission power of the signal source.

In the second time slot, employing DF relay which means that the signal will be forwarded by the relay node only after the decoding is successful. In the system model, $R$ transmits secrecy signal to $D$ and $E$ through the legitimate channel ( $R \rightarrow D$ ) and eavesdropping channel ( $R \rightarrow E$ ), respectively. We can express the message received at $D$ or $E$ as

$$
\mathbf{y}_{R i}=\sqrt{P_{R}} \mathbf{h}_{R i} y_{R}+\mathbf{z}_{R i}, \quad i \in\{D, E\},
$$

where the value of $i$ indicates the legitimate link $(i=D)$ or eavesdropping link $(i=E), \mathbf{y}, \mathbf{h}$ and $\mathbf{z}$ are three vectors representing the received signal, channel gain and complex AWGN. $P_{R}$ denotes the transmitted power at the relay node.

On the basis of (1) and (2) and [15], we can obtain the end-to-end signal-to-noise ratio (SNRs) as

$$
\begin{aligned}
& \gamma_{S D}=\sum_{j=1}^{L_{D}} \gamma_{S D_{j}}=\min \left\{L_{D} \gamma_{S R}, \sum_{j=1}^{L_{D}} \gamma_{R D_{j}}\right\}=\min \left\{L_{D} \gamma_{S R}, \gamma_{D}\right\}, \\
& \gamma_{S E}=\sum_{j=1}^{L_{E}} \gamma_{S E_{j}}=\min \left\{L_{E} \gamma_{S R}, \sum_{j=1}^{L_{E}} \gamma_{R E_{j}}\right\}=\min \left\{L_{E} \gamma_{S R}, \gamma_{E}\right\},
\end{aligned}
$$

where $\gamma$ is the end-to-end SNR, $L_{D}$ and $L_{E}$ are the number of receiving antennas at $D$ and $E$.

\subsection{Channel Characteristics}

In this subsection, we describe the statistical characteristics of $\kappa-\mu$ shadowed distribution. In the system model considered, the channel of $S \rightarrow R$ is a SISO link. According [30] and [43, Eq. (9.210.1)], the PDF for SNR at $R$ can be written as

$$
f_{S R}(\gamma)=a_{S R}^{\mu_{S R}} b_{S R}{ }^{-m_{S R}} \frac{1}{\Gamma\left(\mu_{S R}\right)} \sum_{q_{3}=0}^{\infty} \frac{\left(m_{S R}\right)_{q_{3}}}{\left(\mu_{S R}\right)_{q_{3}} q_{3} !}\left(\frac{a_{S R} k_{S R} \mu_{S R}}{b_{S R} m_{S R}}\right)^{q_{3}} \gamma^{\mu_{S R}+q_{3}-1} \exp \left(-a_{S R} \gamma\right),
$$

where $a_{i}=\mu_{i}\left(1+k_{i}\right) / \Omega_{i}, b_{i}=\mu_{i} k_{i}+m_{i} / m_{i}, k_{i}, \mu_{i}$, and $m_{i}$ are the shape parameters for $\kappa-\mu$ shadowed fading channels. $\Omega_{i}$ means the average SNRs for each channels. $\Gamma(\cdot)$ is defined as the Gamma function [43, Eq. (8.310.1)], and $(x)_{q}$ denotes pochammer operation [43].

For the channels $R \rightarrow D$ and $R \rightarrow E$, there are multiple fading subchannels due to multiantennas reception, and the PDF for the received SNR at $D$ or $E$ can be stated as [44]

$$
\begin{aligned}
f_{i}(\gamma)= & \left(L_{i} a_{i}\right)^{L_{i} \mu_{i}}\left(b_{i}\right)^{-L m_{i}} \frac{1}{\Gamma\left(L_{i} \mu_{i}\right)} \\
& \times \sum_{q=0}^{\infty} \frac{\left(L_{i} m_{i}\right)_{q}}{\left(L_{i} \mu_{i}\right)_{q} q !}\left(\frac{L_{i} a_{i} k_{i} \mu_{i}}{b_{i} m_{i}}\right)^{q} \gamma^{L_{i} \mu_{i}+q-1} \exp \left(-L_{i} a_{i} \gamma\right), i \in\{D, E\} .
\end{aligned}
$$




\section{SOP Analysis}

As an important metric to evaluate the security capability of WCNs, SOP can be interpreted as the probability that the instantaneous secrecy rate is lower than a predetermined threshold value $C_{t h}$ [45]. From [6], we can obtain SOP as

$$
S O P=P\left\{C_{S} \leq C_{t h}\right\}=P\left\{C_{S D}-C_{S E} \leq C_{t h}\right\}=1-P\left\{C_{S}>C_{t h}\right\},
$$

where $C_{S}=\max \left\{\ln \left(1+\gamma_{S D}\right)-\ln \left(1+\gamma_{S E}\right), 0\right\}, C_{S D}=\ln \left(1+\gamma_{S D}\right)$ and $C_{S E}=\ln \left(1+\gamma_{S E}\right)$ are the instantaneous capacity of legitimate channel and eavesdropping channel, respectively. Combined with (3), (4) and (7), we can divide the calculation of $P\left\{C_{S}>C_{t h}\right\}$ into four cases as

$$
\begin{aligned}
P\left\{C_{S}>C_{t h}\right\} & =P\left\{C_{S D}-C_{S E}>C_{t h}\right\} \\
& =P\left\{1+\min \left(L_{D} \gamma_{S R}, \gamma_{D}\right)>\Theta\left(1+\min \left(L_{E} \gamma_{S R}, \gamma_{E}\right)\right)\right\} \\
& =\Phi_{1}+\Phi_{2}+\Phi_{3}+\Phi_{4},
\end{aligned}
$$

where $\Theta=\exp \left(C_{t h}\right) \geq 1$, and

$$
\begin{aligned}
& \Phi_{1}=P\left\{1+\gamma_{D}>\Theta\left(1+\gamma_{E}\right), L_{D} \gamma_{S R}>\gamma_{D}, L_{E} \gamma_{S R}>\gamma_{E}\right\}, \\
& \Phi_{2}=P\left\{1+\gamma_{D}>\Theta\left(1+L_{E} \gamma_{S R}\right), L_{D} \gamma_{S R}>\gamma_{D}, L_{E} \gamma_{S R}<\gamma_{E}\right\}, \\
& \Phi_{3}=P\left\{1+L_{D} \gamma_{S R}>\Theta\left(1+\gamma_{E}\right), L_{D} \gamma_{S R}<\gamma_{D}, L_{E} \gamma_{S R}>\gamma_{E}\right\}, \\
& \Phi_{4}=P\left\{1+L_{D} \gamma_{S R}>\Theta\left(1+L_{E} \gamma_{S R}\right), L_{D} \gamma_{S R}<\gamma_{D}, L_{E} \gamma_{S R}<\gamma_{E}\right\} .
\end{aligned}
$$

Obviously, referring to (9), (10), (11) and (12), when $L_{D} \leq L_{E}, \Phi_{2}$ and $\Phi_{4}$ are impossible to occur, hence, we have $\Phi_{2}=\Phi_{4}=0$, and $P\left\{C_{S}>C_{t h}\right\}$ can then be simplified as

$$
P\left\{C_{S}>C_{t h}\right\}=\Phi_{1}+\Phi_{3} \text {. }
$$

However, for $L_{D}>L_{E}$, all four cases are possible, and the probability can be written as

$$
P\left\{C_{S}>C_{t h}\right\}=\Phi_{1}+\Phi_{2}+\Phi_{3}+\Phi_{4}
$$

Utilizing (5) and (6), we can derive $\Phi_{1}$ as

$$
\begin{aligned}
\Phi_{1} & =P\left\{\Theta\left(1+\gamma_{E}\right)-1<\gamma_{D}<L_{D} \gamma_{S R}, L_{E} \gamma_{S R}>\gamma_{E}\right\} \\
& =\int_{0}^{\infty} f_{\gamma_{S R}}(x) \int_{0}^{L_{E} x} f_{E}(y) \int_{\Theta(1+y)-1}^{L_{D} x} f_{D}(z) d z d y d x \\
& =\sum_{q_{1}=0}^{\infty} A_{1} \int_{0}^{\infty} f_{\gamma_{S R}}(x) \int_{0}^{L_{E} x} f_{E}(y) \int_{\Theta(1+y)-1}^{L_{D} x} z^{L_{D} \mu_{D}+q_{1}-1} \exp \left(-L_{D} a_{D} z\right) d z d y d x,
\end{aligned}
$$

where

$$
A_{1}=\left(L_{D} a_{D}\right)^{L_{D} \mu_{D}}\left(b_{D}\right)^{-L_{D} m_{D}} \frac{1}{\Gamma\left(L_{D} \mu_{D}\right)} \frac{\left(L_{D} m_{D}\right)_{q_{1}}}{\left(L_{D} \mu_{D}\right)_{q_{1}} q_{1} !}\left(\frac{L_{D} a_{D} k_{D} \mu_{D}}{b_{D} m_{D}}\right)^{q_{1}} .
$$

We let $\Phi_{11}=\int_{\Theta(1+y)-1}^{L_{D} x} z^{L_{D} \mu_{D}+q_{1}-1} \exp \left(-L_{D} a_{D} z\right) d z$, with the aid of [43, Eq. (8.310.1)], [39, Eq. (8.352.6)] and [43, Eq. (1.111)], $\Phi_{11}$ can be expressed as 


$$
\begin{aligned}
\Phi_{11} & =\left(L_{D} a_{D}\right)^{-\left(L_{D} \mu_{D}+q_{1}\right)}\left(L \mu_{D}+q_{1}-1\right) !\left(\exp \left(-L_{D} a_{D}(\Theta y+\Theta-1)\right)\right. \\
& \left.\times \sum_{s 2=0}^{L_{D} \mu_{D}+q_{1}-1} \frac{L_{D}{ }^{s_{2}} a_{D}{ }^{s_{2}} \sum_{t=0}^{s_{2}}\left(\begin{array}{l}
s_{2} \\
t
\end{array}\right) \Theta^{t}(\Theta-1)^{s_{2}-t} y^{t}}{s_{2} !}-\exp \left(-L_{D}{ }^{2} a_{D} x\right) \sum_{s_{1}=0}^{L \mu_{D}+q_{1}-1} \frac{\left(L_{D}{ }^{2} a_{D} X\right)^{s_{1}}}{s_{1} !}\right)
\end{aligned}
$$

Substituting (5), (6) and (17) into (15), after a series of complex integral and algebraic operations, we obtain

$$
\begin{aligned}
\Phi_{1} & =\sum_{a_{3}=0}^{\infty} A_{5} \Delta_{1}\left(L_{E} \mu_{E}+q_{2}+t-1\right) !\left(\frac{\Gamma\left(\mu_{S R}+q_{3}\right)}{\left.a_{S R} \mu_{S R}+q_{3}\right)}-\sum_{m_{1}=0}^{L_{E} \mu_{E}+q_{2}+t-1} \frac{L_{E}{ }^{m_{1}}\left(L_{E} a_{E}+L_{D} a_{D} \Theta\right)^{m_{1}}}{m_{1} !}\right. \\
& \left.\times \frac{\Gamma\left(\mu_{S R}+q_{3}+m_{1}\right)}{\left(a_{S R}+L_{E} L_{E} a_{E}+L_{E} L_{D} a_{D} \Theta\right)^{\left(\mu_{S R}+q_{3}+m_{1}\right)}}\right)-\sum_{q_{3}=0}^{\infty} A_{5} \Delta_{2}\left(L_{E} \mu_{E}+q_{2}-1\right) ! \\
& \times\left(\frac{\Gamma\left(\mu_{S R}+q_{3}+s_{1}\right)}{\left(a_{S R}+L_{D}{ }^{2} a_{D}\right)^{\left(\mu_{S R}+q_{3}+S_{1}\right)}}-\sum_{m_{2}=0}^{L_{E} \mu_{E}+q_{2}-1} \frac{a_{E}^{m_{2}} L_{E}{ }^{2 m_{2}} \Gamma\left(\mu_{S R}+q_{3}+s_{1}+m_{2}\right)}{m_{2} !\left(a_{S R}+L_{D}{ }^{2} a_{D}+L_{E}{ }^{2} a_{E}\right)^{\left(\mu_{S R}+q_{3}+s_{1}+m_{2}\right)}}\right),
\end{aligned}
$$

where

$$
\begin{aligned}
\Delta_{1}= & \sum_{q_{1}=0}^{\infty} A_{3} \sum_{q_{2}=0}^{\infty} A_{2}\left(L_{D} \mu_{D}+q_{1}-1\right) ! \exp \left(-L_{D} a_{D}(\Theta-1)\right) \\
& \times \sum_{s_{2}=0}^{L \mu_{L_{1}+q_{1}-1}} \frac{L_{D}{ }^{s_{2}} a_{D}{ }^{s_{2}}}{s_{2} !} \sum_{t=0}^{s_{2}}\left(\begin{array}{l}
s_{2} \\
t
\end{array}\right) \Theta^{t}(\Theta-1)^{s_{2}-t}\left(L_{E} a_{E}+L_{D} a_{D} \Theta\right)^{-\left(L_{E} \mu_{E}+q_{2}+t\right)} \\
\Delta_{2}= & \sum_{q_{1}=0}^{\infty} A_{3} \sum_{q_{2}=0}^{\infty} A_{4}\left(L \mu_{D}+q_{1}-1\right) ! \sum_{s_{1}=0}^{L \mu_{D}+q_{1}-1} \frac{\left(L_{D}{ }^{2} a_{D}\right)^{s_{1}}}{s_{1} !}
\end{aligned}
$$

and

$$
\begin{aligned}
& A_{2}=\left(L_{E} a_{E}\right)^{L_{E} \mu_{E}} b_{E}^{-L_{E} m_{E}} \frac{\left(L_{E} m_{E}\right)_{q_{2}}}{\Gamma\left(L_{E} \mu_{E}\right)\left(L_{E} \mu_{E}\right)_{q_{2}} q_{2} !}\left(\frac{L_{E} a_{E} k_{E} \mu_{E}}{b_{E} m_{E}}\right)^{q_{2}}, \\
& A_{3}=\left(L_{D} a_{D}\right)^{-q_{1}} b_{D}{ }^{-L_{D} m_{D}} \frac{\left(L_{D} m_{D}\right)_{q_{1}}}{\Gamma\left(L_{D} \mu_{D}\right)\left(L_{D} \mu_{D}\right)_{q_{1}} q_{1} !}\left(\frac{L_{D} a_{D} k_{D} \mu_{D}}{b_{D} m_{D}}\right)^{q_{1}}, \\
& A_{4}=\left(L_{E} a_{E}\right)^{-q_{2}} b_{E}{ }^{-L_{E} m_{E}} \frac{\left(L_{E} m_{E}\right)_{q_{2}}}{\Gamma\left(L_{E} \mu_{E}\right)\left(L_{E} \mu_{E}\right)_{q_{2}} q_{2} !}\left(\frac{L_{E} a_{E} k_{E} \mu_{E}}{b_{E} m_{E}}\right)^{q_{2}}, \\
& A_{5}=a_{S R}{ }^{\mu_{S R}} b_{S R}{ }^{-m_{S R}} \frac{1}{\Gamma\left(\mu_{S R}\right)}\left(\frac{a_{S R} k_{S R} \mu_{S R}}{b_{S R} m_{S R}}\right)^{q_{3}} \frac{\left(m_{S R}\right)_{q_{3}}}{\left(\mu_{S R}\right)_{q_{3}} q_{3} !}
\end{aligned}
$$

After analyzing (10), we obtain the expanded expression as

$$
\begin{aligned}
\Phi_{2} & =\operatorname{Pr}\left\{\Theta\left(1+L_{E} \gamma_{S R}\right)-1<\gamma_{D}<L_{D} \gamma_{S R}, \gamma_{E}>L_{E} \gamma_{S R}\right\} \\
& =\int_{0}^{\infty} f_{\gamma_{S R}}(x) \int_{L_{E} x}^{\infty} f_{E}(y) \int_{\Theta\left(1+L_{E} x\right)-1}^{L_{D} x} f_{D}(z) d z d y d x .
\end{aligned}
$$

With the help of (5) , (6), [43, Eq. (8.310.1)], [43, Eq. (8.352.6)] and [43, Eq. (1.111)], we can gradually expand the integral, and the final expression of $\Phi_{2}$ is as 
where

$$
\Phi_{2}=\Phi_{21}-\Phi_{22}-\Phi_{23}+\Phi_{24}+\Phi_{25}-\Phi_{26}
$$

$$
\begin{aligned}
& \Phi_{21}=\sum_{q_{3}=0}^{\infty} A_{5} \sum_{q_{1}=0}^{\infty} A_{3} \sum_{q_{2}=0}^{\infty} A_{2}\left(L_{D} \mu_{D}+q_{1}-1\right) ! \frac{\Gamma\left(L_{E} \mu_{E}+q_{2}\right)}{\left(L_{E} a_{E}\right)^{\left(L_{E} \mu_{E}+q_{2}\right)}} \exp \left(-L_{D} a_{D}(\Theta-1)\right) \\
& \times \sum_{s 2=0}^{L_{D} \mu_{D}+q_{1}-1} \frac{\left(L_{D} a_{D}\right)^{s_{2}}}{s_{2} !} \sum_{t=0}^{s_{2}}\left(\begin{array}{l}
s_{2} \\
t
\end{array}\right)\left(\Theta L_{E}\right)^{t}(\Theta-1)^{s_{2}-t} \frac{\Gamma\left(\mu_{S R}+q_{3}+t\right)}{\left(a_{S R}+L_{D} a_{D} \Theta L_{E}\right)^{\mu_{S R}+q_{3}+t}}, \\
& \Phi_{22}=\sum_{q_{3}=0}^{\infty} A_{5} \sum_{q_{1}=0}^{\infty} A_{3} \sum_{q_{2}=0}^{\infty} A_{2}\left(L_{D} \mu_{D}+q_{1}-1\right) ! \frac{\Gamma\left(L_{E} \mu_{E}+q_{2}\right)}{\left(L_{E} a_{E}\right)^{\left(L_{E} \mu_{E}+q_{2}\right)}} \\
& \times \sum_{s_{1}=0}^{L_{D} \mu_{D}+a_{1}-1} \frac{\left(L_{D}{ }^{2} a_{D}\right)^{s_{1}}}{s_{1} !} \frac{\Gamma\left(\mu_{S R}+q_{3}+s_{1}\right)}{\left(a_{S R}+L_{D}{ }^{2} a_{D}\right)^{\mu_{S R}+q_{3}+s_{1}}}, \\
& \Phi_{23}=\sum_{q_{3}=0}^{\infty} A_{5} \sum_{q_{1}=0}^{\infty} A_{3} \sum_{q_{2}=0}^{\infty} A_{4}\left(L_{D} \mu_{D}+q_{1}-1\right) !\left(L_{E} \mu_{E}+q_{2}-1\right) ! \exp \left(-L_{D} a_{D}(\Theta-1)\right) \\
& \times \sum_{s_{2}=0}^{L_{D} \mu_{D}+q_{1}-1} \frac{\left(L_{D} a_{D}\right)^{s_{2}}}{s_{2} !} \sum_{t=0}^{s_{2}}\left(\begin{array}{l}
s_{2} \\
t
\end{array}\right)\left(\Theta L_{E}\right)^{t}(\Theta-1)^{s_{2}-t} \frac{\Gamma\left(\mu_{S R}+q_{3}+t\right)}{\left(a_{S R}+L_{D} a_{D} \Theta L_{E}\right)^{\mu_{S R}+q_{3}+t}}, \\
& \Phi_{24}=\sum_{q_{3}=0}^{\infty} A_{5} \sum_{q_{1}=0}^{\infty} A_{3} \sum_{q_{2}=0}^{\infty} A_{4}\left(L_{D} \mu_{D}+q_{1}-1\right) !\left(L_{E} \mu_{E}+q_{2}-1\right) ! \\
& \times \sum_{s_{1}=0}^{L_{D} \mu_{D}+q_{1}-1} \frac{\left(L_{D}^{2} a_{D}\right)^{s_{1}}}{s_{1} !} \frac{\Gamma\left(\mu_{S R}+q_{3}+s_{1}\right)}{\left(a_{S R}+L_{D}^{2} a_{D}\right)^{\mu_{S R}+q_{3}+s_{1}}}, \\
& \Phi_{25}=\sum_{q_{3}=0}^{\infty} A_{5} \sum_{q_{1}=0}^{\infty} A_{3} \sum_{q_{2}=0}^{\infty} A_{4}\left(L_{D} \mu_{D}+q_{1}-1\right) !\left(L_{E} \mu_{E}+q_{2}-1\right) ! \exp \left(-L_{D} a_{D}(\Theta-1)\right) \\
& \times \sum_{s_{2}=0}^{L_{D} \mu_{D}+q_{1}-1} \frac{\left(L_{D} a_{D}\right)^{s_{2}}}{s_{2} !} \sum_{t=0}^{s_{2}}\left(\begin{array}{l}
s_{2} \\
t
\end{array}\right)\left(\Theta L_{E}\right)^{t}(\Theta-1)^{s_{2}-t} \\
& \times \sum_{m_{1}=0}^{L_{E} \mu_{E}+q_{2}-1} \frac{\left(L_{E}{ }^{2} a_{E}\right)^{m_{1}}}{m_{1} !} \frac{\Gamma\left(\mu_{S R}+q_{3}+t+m_{1}\right)}{\left(a_{S R}+L_{D} a_{D} \Theta L_{E}+L_{E}^{2} a_{E}\right)^{\mu_{S R}+q_{3}+t+m_{1}}}, \\
& \Phi_{26}=\sum_{q_{3}=0}^{\infty} A_{5} \sum_{q_{1}=0}^{\infty} A_{3} \sum_{q_{2}=0}^{\infty} A_{4}\left(L_{D} \mu_{D}+q_{1}-1\right) !\left(L_{E} \mu_{E}+q_{2}-1\right) ! \\
& \times \sum_{s_{1}=0}^{L_{D} \mu_{D}+q_{1}-1} \frac{\left(L_{D}{ }^{2} a_{D}\right)^{s_{1}}}{s_{1} !} \sum_{m_{1}=0}^{L_{E} \mu_{E}+q_{2}-1} \frac{\left(L_{E}{ }^{2} a_{E}\right)^{m_{1}}}{m_{1} !} \frac{\Gamma\left(\mu_{S R}+q_{3}+s_{1}+m_{1}\right)}{\left(a_{S R}+L_{D}{ }^{2} a_{D}+L_{E}{ }^{2} a_{E}\right)^{\mu_{S R}+q_{3}+s_{1}+m_{1}}} .
\end{aligned}
$$

For the third case, the calculation of $\Phi_{3}$ can be expressed as 


$$
\begin{aligned}
\Phi_{3} & =\operatorname{Pr}\left\{1+L_{D} \gamma_{S R}>\Theta\left(1+\gamma_{E}\right), L_{D} \gamma_{S R}<\gamma_{D}, L_{E} \gamma_{S R}>\gamma_{E}\right\} \\
& =\operatorname{Pr}\left\{\gamma_{E}<\frac{1+L_{D} \gamma_{S R}-\Theta}{\Theta}, L_{D} \gamma_{S R}<\gamma_{D}\right\} \\
& =\int_{0}^{\infty} f_{\gamma_{S R}}(x) \int_{0}^{\frac{1+L_{D} x-\Theta}{\Theta}} f_{E}(y) \int_{L_{D} x}^{\infty} f_{D}(z) d z d y d x \\
& =\int_{0}^{\infty} f_{\gamma_{S R}}(x) \int_{0}^{\frac{1+L_{D} x-\Theta}{\Theta}} f_{E}(y)\left(\int_{0}^{\infty} f_{D}(z) d z-\int_{0}^{L_{D} x} f_{D}(z) d z\right) d y d x .
\end{aligned}
$$

In (33), $\Phi_{3}$ contains triple integrals, similar to the derivation of $\Phi_{1}$, and making use of [43, Eq. (3.326.2)], we obtain the expression of $\Phi_{3}$ as

$$
\Phi_{3}=\sum_{q_{3}=0}^{\infty} A_{5} \sum_{q_{1}=0}^{\infty} A_{1} \sum_{q_{2}=0}^{\infty} A_{4}\left(L_{E} \mu_{E}+q_{2}-1\right) !\left(\Phi_{31}-\Phi_{32}\right),
$$

where

$$
\begin{aligned}
& \Phi_{31}=\left(\left(\frac{\Gamma\left(L_{D} \mu_{D}+q_{1}\right)}{\left(L_{D} a_{D}\right)^{\left(L_{D} \mu_{D}+q_{1}\right)}}-\left(L_{D} a_{D}\right)^{-\left(L_{D} \mu_{D}+q_{1}\right)}\left(L_{D} \mu_{D}+q_{1}-1\right) !\right) \frac{\Gamma\left(\mu_{S R}+q_{3}\right)}{a_{S R}{ }^{\left(\mu_{S R}+q_{3}\right)}}\right. \\
&\left.+\left(L_{D} a_{D}\right)^{-\left(L_{D} \mu_{D}+q_{1}\right)}\left(L_{D} \mu_{D}+q_{1}-1\right) ! \sum_{s_{1}=0}^{L_{D} \mu_{D}+q_{1}-1} \frac{\left(L_{D}{ }^{2} a_{D}\right)^{s_{1}} \Gamma\left(\mu_{S R}+q_{3}+s_{1}\right)}{\left.s_{1} !\left(a_{S R}+L_{D}{ }^{2} a_{D}\right)^{\left(\mu_{S R}+q_{3}+s_{1}\right)}\right)}\right), \\
& \Phi_{32}= \exp \left(\frac{L_{E} a_{E}(\Theta-1)}{\Theta}\right) \sum_{s_{2}=0}^{L_{E} \mu_{E}+q_{2}-1} \frac{\left(L_{E} a_{E}\right)^{s_{2}}}{\Theta^{s_{2}} s_{2} !} \sum_{t=0}^{s_{2}}\left(\begin{array}{l}
s_{2} \\
t
\end{array}\right) L_{D}{ }^{t}(1-\Theta)^{s_{2}-t}\left(\left(\frac{\Gamma\left(L_{D} \mu_{D}+q_{1}\right)}{\left(L_{D} a_{D}\right)^{\left(L_{D} \mu_{D}+q_{1}\right)}}\right.\right. \\
&\left.-\left(L_{D} a_{D}\right)^{-\left(L_{D} \mu_{D}+q_{1}\right)}\left(L_{D} \mu_{D}+q_{1}-1\right) !\right) \frac{\Gamma\left(\mu_{S R}+q_{3}+t\right)}{\left(a_{S R}+\frac{L_{E} a_{E} L_{D}}{\Theta}\right)^{\left(\mu_{S R}+q_{3}+t\right)}+\left(L_{D} a_{D}\right)^{-\left(L_{D} \mu_{D}+q_{1}\right)}} \\
& \times\left(L_{D} \mu_{D}+q_{1}-1\right) ! \\
&\left.\sum_{s_{1}=0}^{L_{D} \mu_{D}+q_{1}-1} \frac{\left(L_{D}{ }^{2} a_{D}\right)^{s_{1}} \Gamma\left(\mu_{S R}+q_{3}+t+s_{1}\right)}{s_{1} !\left(a_{S R}+\frac{L_{E} a_{E} L_{D}}{\Theta}+L_{D}{ }^{2} a_{D}\right)^{\left(\mu_{S R}+q_{3}+t+s_{1}\right)}}\right) .
\end{aligned}
$$

Similarly, we have

$$
\begin{aligned}
\Phi_{4}= & \sum_{q 3=0}^{\infty} A_{5} \sum_{q_{1}=0}^{\infty} A_{1} \sum_{q_{2}=0}^{\infty} A_{2} \sum_{s_{1}=0}^{L_{D} \mu_{D}+q_{1}-1} \frac{\left(L_{D} \mu_{D}+q_{1}-1\right) ! L_{D} s_{1}}{s_{1} !\left(L_{D} a_{D}\right)^{L_{D} \mu_{D}+q_{1}-s_{1}}} \\
& \times \sum_{s_{2}=0}^{L_{E} \mu_{E}+q_{2}-1} \frac{\left(L_{E} \mu_{E}+q_{2}-1\right) ! L_{E}^{s_{2}}\left(a_{S R}+L_{D}{ }^{2} a_{D}+L_{E}^{2} a_{E}\right)^{-\left(\mu_{S R}+q_{3}+s_{1}+s_{2}\right)}}{s_{2} !\left(L_{E} a_{E}\right)^{L_{E} \mu_{E}+q_{2}-s_{2}}} \\
& \times \Gamma\left(\mu_{S R}+q_{3}+s_{1}+s_{2}, \frac{\left(a_{S R}+L_{D}{ }^{2} a_{D}+L_{E}{ }^{2} a_{E}\right)(\Theta-1)}{L_{D}-\Theta L_{E}}\right) .
\end{aligned}
$$

According to (7), (18), (25), (34) and (37), under the premise that $L_{D} \leq L_{E}$ exists, we finally express SOP as

$$
S O P=1-\left(\Phi_{1}+\Phi_{3}\right)
$$


When $L_{D}>L_{E}$ is satisfied, the SOP can be expressed as

$$
S O P=1-\left(\Phi_{1}+\Phi_{2}+\Phi_{3}+\Phi_{4}\right) .
$$

\section{SPSC Analysis}

In this section, we discuss another fundamental criterion in wireless environments, i.e., SPSC, which is defined as the probability of taking positive value of instantaneous safety capacity in [45]. The SPSC can be expressed as

$$
\begin{aligned}
S P S C & =P\left\{C_{S}\left(\gamma_{D}, \gamma_{E}\right)>0\right\} \\
& =P\left\{1+\min \left(L_{D} \gamma_{S R}, \gamma_{D}\right)>1+\min \left(L_{E} \gamma_{S R}, \gamma_{E}\right)\right\} \\
& =P\left\{\min \left(L_{D} \gamma_{S R}, \gamma_{D}\right)>\min \left(L_{E} \gamma_{S R}, \gamma_{E}\right)\right\} .
\end{aligned}
$$

For the relationship of $\left(L_{D} \gamma_{S R}, \gamma_{D}\right)$ and $\left(L_{E} \gamma_{S R}, \gamma_{E}\right)$, we can explain it in four cases: i) $L_{D} \gamma_{S R}>\gamma_{D}, L_{E} \gamma_{S R}>\gamma_{E}$; ii) $L_{D} \gamma_{S R}>\gamma_{D}, L_{E} \gamma_{S R}<\gamma_{E}$; iii) $L_{D} \gamma_{S R}<\gamma_{D}, L_{E} \gamma_{S R}>\gamma_{E}$; iv) $L_{D} \gamma_{S R}<\gamma_{D}, L_{E} \gamma_{S R}<\gamma_{E}$. It should be noted that he second and fourth cases are impossible events under the condition of $L_{D} \leq L_{E}$. But if $L_{D}>L_{E}$, all four cases are necessary.

The integral expansion of $I_{1}$ is

$$
\begin{aligned}
I_{1} & =P\left\{L_{D} \gamma_{S R}>\gamma_{D}>\gamma_{E}, \gamma_{E}<L_{E} \gamma_{S R}\right\} \\
& =\int_{0}^{\infty} f_{\gamma_{S R}}(x) \int_{0}^{L_{E} x} f_{E}(y) \int_{y}^{L_{D} x} f_{D}(z) d z d y d x .
\end{aligned}
$$

With the help of (5), (6), [43, Eq. (8.310.1)] and [43, Eq. (8.352.6)], $I_{1}$ is derived as

$$
\begin{aligned}
I_{1}= & \sum_{q_{3}=0}^{\infty} A_{5} \Psi_{1}\left(L_{E} \mu_{E}+q_{2}+s_{2}-1\right) ! \\
& \times\left(\frac{\Gamma\left(\mu_{S R}+q_{3}\right)}{a_{S R}{ }^{\left(\mu_{S R}+q_{3}\right)}}-\sum_{m_{1}=0}^{L_{E} \mu_{E}+q_{2}+s_{2}-1} \frac{L_{E}{ }^{m_{1}}\left(L_{E} a_{E}+L_{D} a_{D}\right)^{m_{1}} \Gamma\left(\mu_{S R}+q_{3}+m_{1}\right)}{\left.m_{1} !\left(a_{S R}+L_{E} L_{E} a_{E}+L_{E} L_{D} a_{D}\right)^{\left(\mu_{S R}+q_{3}+m_{1}\right)}\right)}\right. \\
& -\sum_{q_{3}=0}^{\infty} A_{5} \Psi_{2}\left(L_{E} \mu_{E}+q_{2}-1\right) ! \\
& \times\left(\frac{\Gamma\left(\mu_{S R}+q_{3}+s_{1}\right)}{\left(a_{S R}+L_{D}{ }^{2} a_{D}\right)^{\left(\mu_{S R}+q_{3}+s_{1}\right)}}-\sum_{m_{2}=0}^{L_{E} \mu_{E}+q_{2}-1} \frac{\left(L_{E}{ }^{2} a_{E}\right)^{m_{2}} \Gamma\left(\mu_{S R}+q_{3}+s_{1}+m_{2}\right)}{m_{2} !\left(a_{S R}+L_{D}{ }^{2} a_{D}+L_{E}{ }^{2} a_{E}\right)^{\left(\mu_{S R}+q_{3}+s_{1}+m_{2}\right)}}\right),
\end{aligned}
$$

where

$$
\Psi_{1}=\sum_{q_{1}=0}^{\infty} A_{3} \sum_{q_{2}=0}^{\infty} A_{2}\left(L_{D} \mu_{D}+q_{1}-1\right) ! \sum_{s_{2}=0}^{L_{D} \mu_{D}+q_{1}-1} \frac{L_{D}{ }^{s_{2}} a_{D}{ }^{s_{2}}}{s_{2} !}\left(L_{E} a_{E}+L_{D} a_{D}\right)^{-\left(L_{E} \mu_{E}+q_{2}+s_{2}\right)},
$$

and

$$
\Psi_{2}=\sum_{q_{1}=0}^{\infty} A_{3} \sum_{q_{2}=0}^{\infty} A_{4}\left(L_{D} \mu_{D}+q_{1}-1\right) ! \sum_{s_{1}=0}^{L_{D} \mu_{D}+q_{1}-1} \frac{\left(L_{D}{ }^{2} a_{D}\right)^{s_{1}}}{s_{1} !} .
$$

Similar to the derivation of $I_{1}, I_{2}$ is obtained as 


$$
\begin{aligned}
I_{2}= & P\left\{L_{D} \gamma_{S R}>\gamma_{D}>L_{E} \gamma_{S R}, \gamma_{E}<L_{E} \gamma_{S R}\right\} \\
= & \sum_{q_{3}=0}^{\infty} A_{5} \sum_{q_{1}=0}^{\infty} A_{3} \sum_{q_{2}=0}^{\infty} A_{4}\left(L_{D} \mu_{D}+q_{1}-1\right) !\left(L_{E} \mu_{E}+q_{2}-1\right) ! \\
& \times\left(\left(\sum_{s 2=0}^{L_{D} \mu_{D}+q_{1}-1} \frac{\left(L_{D} a_{D} L_{E}\right)^{s_{2}} \Gamma\left(\mu_{S R}+q_{3}+s_{2}\right)}{s_{2} !\left(a_{S R}+L_{D} a_{D} L_{E}\right)^{\left(\mu_{S R}+q_{3}+s_{2}\right)}-} \sum_{s_{1}=0}^{L_{D} \mu_{D}+a_{1}-1} \frac{\left(L_{D}{ }^{2} a_{D}\right)^{s_{1}} \Gamma\left(\mu_{S R}+q_{3}+s_{1}\right)}{s_{1} !\left(a_{S R}+L_{D}{ }^{2} a_{D}\right)^{\left(\mu_{S R}+q_{3}+s_{1}\right)}}\right)\right. \\
& -\left(\sum_{s_{3}=0}^{L_{E} \mu_{E}+q_{2}-1} \frac{\left(L_{E}{ }^{2} a_{E}\right)^{s_{3}}}{s_{3} !} \sum_{s_{D}=0}^{L_{D} \mu_{D}+q_{1}-1} \frac{\left(L_{D} a_{D} L_{E}\right)^{s_{2}} \Gamma\left(\mu_{S R}+q_{3}+s_{3}+s_{2}\right)}{s_{2} !\left(a_{S R}+L_{E}{ }^{2} a_{E}+L_{D} a_{D} L_{E}\right)^{\left(\mu_{S R}+q_{3}+s_{3}+s_{2}\right)}}\right. \\
& \left.\left.-\sum_{s_{3}=0}^{L_{E} \mu_{E}+q_{2}-1} \frac{\left(L_{E}{ }^{2} a_{E}\right)^{s_{3}}}{s_{3} !} \sum_{s_{1}=0}^{L_{D} \mu_{D}+q_{1}-1} \frac{\left(L_{D}{ }^{2} a_{D}\right)^{s_{1}} \Gamma\left(\mu_{S R}+q_{3}+s_{3}+s_{1}\right)}{s_{1} !\left(a_{S R}+L_{D}{ }^{2} a_{D}+L_{E}{ }^{2} a_{E}\right)^{\left(\mu_{S R}+q_{3}+s_{3}+s_{1}\right)}}\right)\right)
\end{aligned}
$$

$I_{3}$ can be written as

$$
\begin{aligned}
& I_{3}=P\left\{\gamma_{E}<L_{E} \gamma_{S R}, L_{D} \gamma_{S R}<\gamma_{D}\right\} \\
& =\sum_{q_{3}=0}^{\infty} A_{5} \sum_{q_{1}=0}^{\infty} A_{1} \sum_{q_{2}=0}^{\infty} A_{4}\left(L_{E} \mu_{E}+q_{2}-1\right) ! \\
& \times\left(\left(\frac{\Gamma\left(L_{D} \mu_{D}+q_{1}\right)}{\left(L_{D} a_{D}\right)^{\left(L_{D} \mu_{D}+q_{1}\right)}}-A_{6}\right) \frac{\Gamma\left(\mu_{S R}+q_{3}\right)}{\left.a_{S R} \mu_{S R}+q_{3}\right)}+A_{6} \sum_{s_{1}=0}^{L_{D} \mu_{D}+q_{1}-1} \frac{\left(L_{D}{ }^{2} a_{D}\right)^{s_{1}} \Gamma\left(\mu_{S R}+q_{3}+s_{1}\right)}{s_{1} !\left(a_{S R}+L_{D}{ }^{2} a_{D}\right)^{\left(\mu_{S R}+q_{3}+s_{1}\right)}}\right. \\
& -\sum_{s_{2}=0}^{L_{E} \mu_{E}+q_{2}-1} \frac{\left(L_{E}{ }^{2} a_{E}\right)^{s_{2}}}{s_{2} !}\left(\frac{\Gamma\left(L_{D} \mu_{D}+q_{1}\right)}{\left(L_{D} a_{D}\right)^{\left(L_{D} \mu_{D}+q_{1}\right)}}-A_{6}\right) \frac{\Gamma\left(\mu_{S R}+q_{3}+s_{2}\right)}{\left(a_{S R}+L_{E}{ }^{2} a_{E}\right)^{\left(\mu_{\text {SR }}+q_{3}+s_{2}\right)}} \\
& \left.+A_{6} \sum_{s_{1}=0}^{L_{D} \mu_{D}+q_{1}-1} \frac{\left(L_{D}{ }^{2} a_{D}\right)^{s_{1}} \Gamma\left(\mu_{S R}+q_{3}+s_{2}+s_{1}\right)}{s_{1} !\left(a_{S R}+L_{E}{ }^{2} a_{E}+L_{D}{ }^{2} a_{D}\right)^{\left(\mu_{S R}+q_{3}+s_{2}+s_{1}\right)}}\right),
\end{aligned}
$$

where

The expression of $I_{4}$ is

$$
A_{6}=\left(L_{D} a_{D}\right)^{-\left(L_{D} \mu_{D}+q_{1}\right)}\left(L_{D} \mu_{D}+q_{1}-1\right) !
$$

$$
\begin{aligned}
I_{4}= & P\left\{\gamma_{E}<L_{E} \gamma_{S R}, \gamma_{D}<L_{D} \gamma_{S R}\right\} \\
= & \sum_{q_{1}=0}^{\infty} A_{3} \sum_{q_{2}=0}^{\infty} A_{4}\left(L_{D} \mu_{D}+q_{1}-1\right) !\left(L_{E} \mu_{E}+q_{2}-1\right) ! \\
& \times\left(\left(\frac{\Gamma\left(\mu_{S R}+q_{3}\right)}{a_{S R} \mu_{S R}+q_{3}}-\sum_{s_{1}=0}^{L_{D} \mu_{D}+q_{1}-1} \frac{\left(L_{D}{ }^{2} a_{D}\right)^{S_{1}} \Gamma\left(\mu_{S R}+q_{3}+s_{1}\right)}{s_{1} !\left(a_{S R}+L_{D}{ }^{2} a_{D}\right)^{\mu_{S R}+q_{3}}}\right)-\sum_{s_{2}=0}^{L_{E} \mu_{E}+q_{2}-1} \frac{\left(L_{E}{ }^{2} a_{E}\right)^{s_{2}}}{s_{2} !}\right. \\
& \left.\times\left(\frac{\Gamma\left(\mu_{S R}+q_{3}+s_{2}\right)}{\left(a_{S R}+L_{E}{ }^{2} a_{E}\right)^{\mu_{S R}+q_{3}+s_{2}}}-\sum_{S_{1}=0}^{L_{D} \mu_{D}+q_{1}-1} \frac{\left(L_{D}{ }^{2} a_{D}\right)^{S_{1}} \Gamma\left(\mu_{S R}+q_{3}+s_{1}+s_{2}\right)}{s_{1} !\left(a_{S R}+L_{D}{ }^{2} a_{D}+L_{E}{ }^{2} a_{E}\right)^{\mu_{S R}+q_{3}+s_{1}+s_{2}}}\right)\right) .
\end{aligned}
$$

Based on (40), (42), (45), (46) and (48), we deduce the closed-form expressions for SPSC 
under two kinds of multi-antenna conditions: i) $S P S C=I_{1}+I_{3}, L_{D} \leq L_{E}$; $\quad$ ii) $S P S C=I_{1}+I_{2}+I_{3}+I_{4}, L_{D}>L_{E}$.

\section{Numerical results}

In this section, we provide some numerical examples of the analytical expressions derived above and Monte Carlo simulations. From the simulation results illustrated in Figs. 2-11, we can see that the theoretical and simulated results are well coincident and the secrecy performance improves with the increase of $\Omega_{S R}$ which represents the average SNR of link $S \rightarrow R$. It should be noted that same parameters in all simulations are set as follows: $L_{D}=L_{E}=L$ in Figs. 2-9, $C_{t h}=0.01 \mathrm{~dB}, \Omega_{S R}=\Omega_{D_{j}}$ and $\Omega_{E_{j}}=10 \mathrm{~dB}$, where $j=0,1, \cdots, L$. In addition, the number of pseudo-random variables of channel gain is $10^{6}$, and after simulation verification, the condition of infinite series convergence is that the value of cyclic variable is more than 60 times. It should be noted that in the following figures, under the same abscissa, lower SOP and higher SPSC mean good security performance, and vice versa.

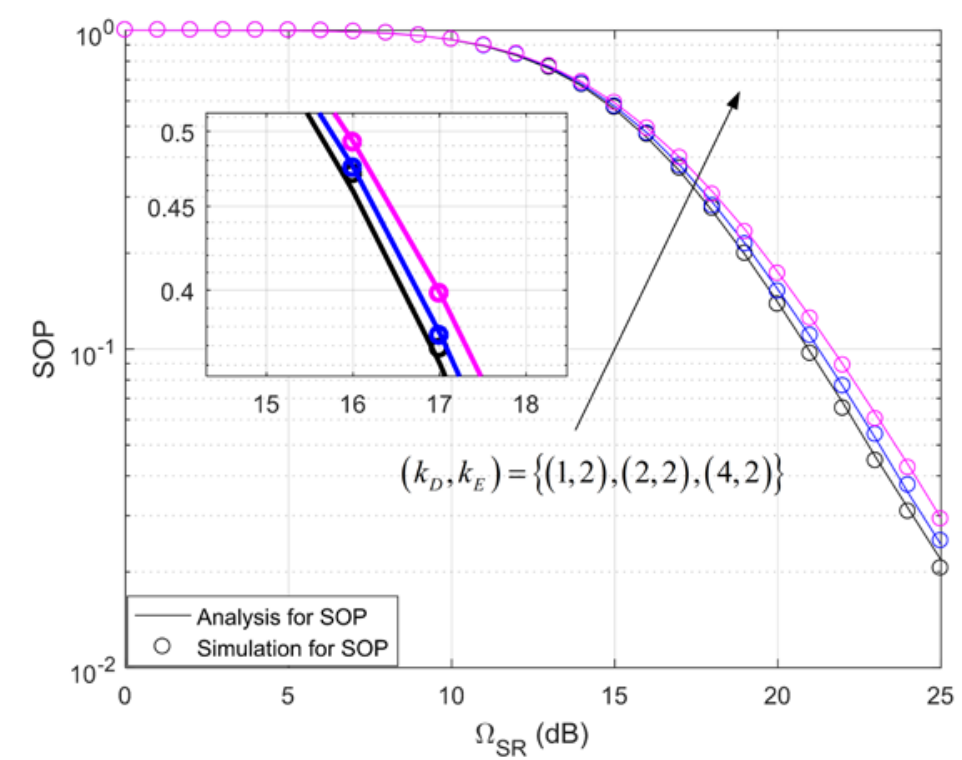

Fig. 2. SOP versus $\Omega_{S R}$ for various values of $\left(k_{D}, k_{E}\right)$ when $\mu_{D}=\mu_{E}=2, m_{D}=m_{E}=1, L=2$. 


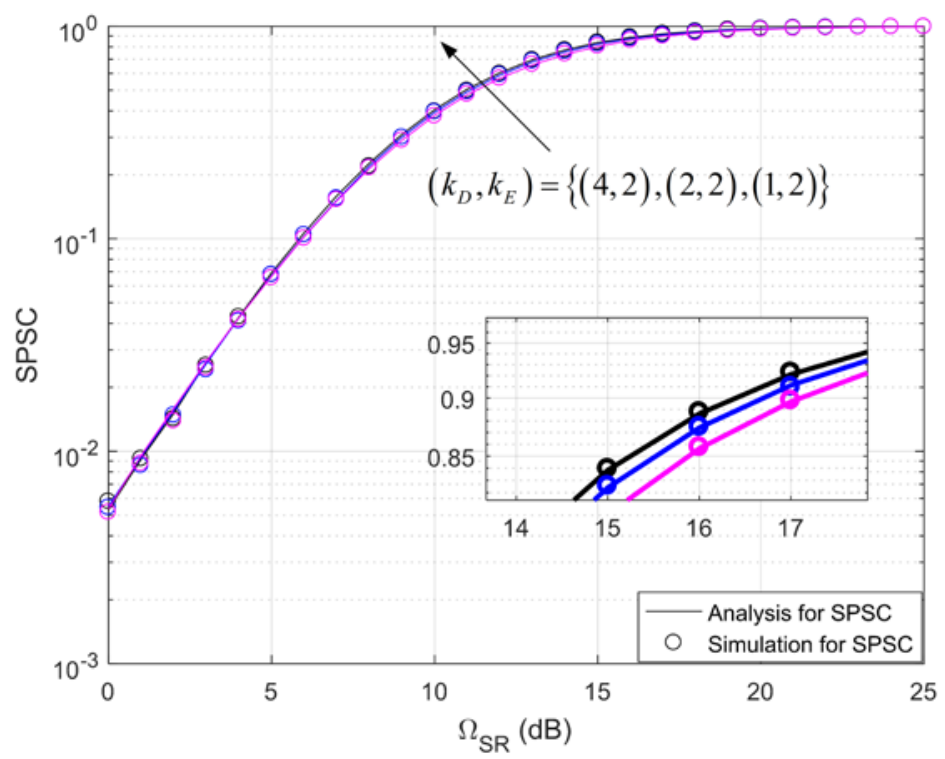

Fig. 3. SPSC versus $\Omega_{S R}$ for various values of $\left(k_{D}, k_{E}\right)$ when $\mu_{D}=\mu_{E}=2, m_{D}=m_{E}=1, L=2$.

Fig. 2 and Fig. 3 depict the curves of SOP and SPSC versus $\Omega_{S R}$ for different $\left(k_{D}, k_{E}\right)$ that $k_{i}$ denote the ratio of the total energy between the main wave and the scattering components in the legitimate link and wiretapping link. When $\Omega_{S R}>10 \mathrm{~dB}$, the larger ratio of $\left(k_{D}, k_{E}\right)$ corresponds to the higher value of SOP and the lower value of SPSC. However, the change of SPSC is not obvious compared with that of SOP. To sum up, under the premise of constant $k_{E}$ and larger $\Omega_{S R}$, large $k_{D}$ will damage the security performance of the relay model under consideration .

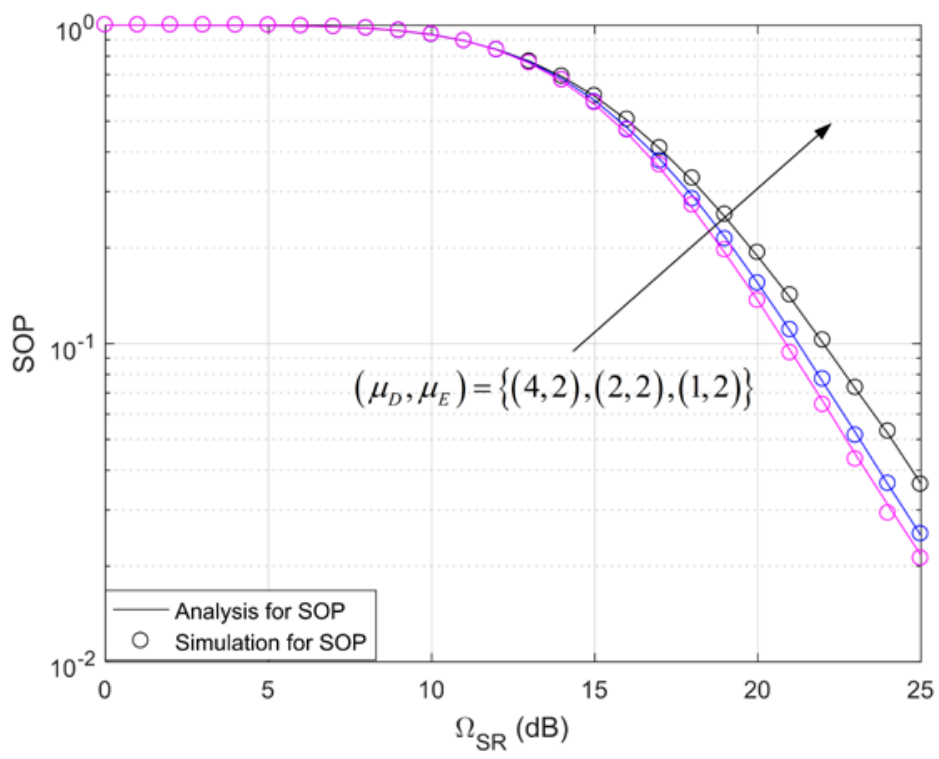

Fig. 4. SOP versus $\Omega_{S R}$ for various values of $\left(\mu_{D}, \mu_{E}\right)$ when $k_{D}=k_{E}=2, m_{D}=m_{E}=1, L=2$. 


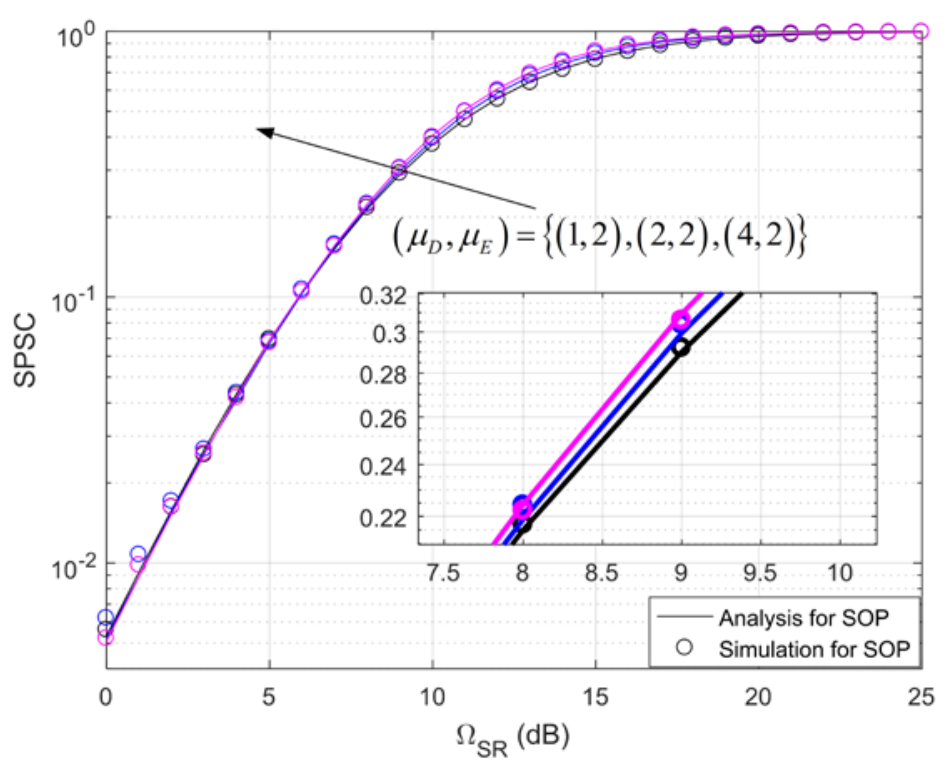

Fig. 5. SPSC versus $\Omega_{S R}$ for various values of $\left(\mu_{D}, \mu_{E}\right)$ when $k_{D}=k_{E}=2, m_{D}=m_{E}=1, L=2$.

Given by Fig. 4, the SOP versus $\Omega_{S R}$ for various values of $\left(\mu_{D}, \mu_{E}\right)$ is provided, where $\mu_{D}$ and $\mu_{E}$ are the quantity for multipath clusters of the legitimate link and wiretapping link. We find that the SOP increases gradually with the decrease of $\mu_{D}$ when $\Omega_{S R}>10 \mathrm{~dB}$. When $\Omega_{S R}<10 \mathrm{~dB}$, the values of SOP and SPSC have barely changed. From Fig. 5 , it can be seen that the increase of $\mu_{D}$ will lead to an increasing SPSC. Therefore, we obtain that the improvement of security performance needs large ratio of $\left(\mu_{D}, \mu_{E}\right)$ when $\Omega_{S R}>10 \mathrm{~dB}$.

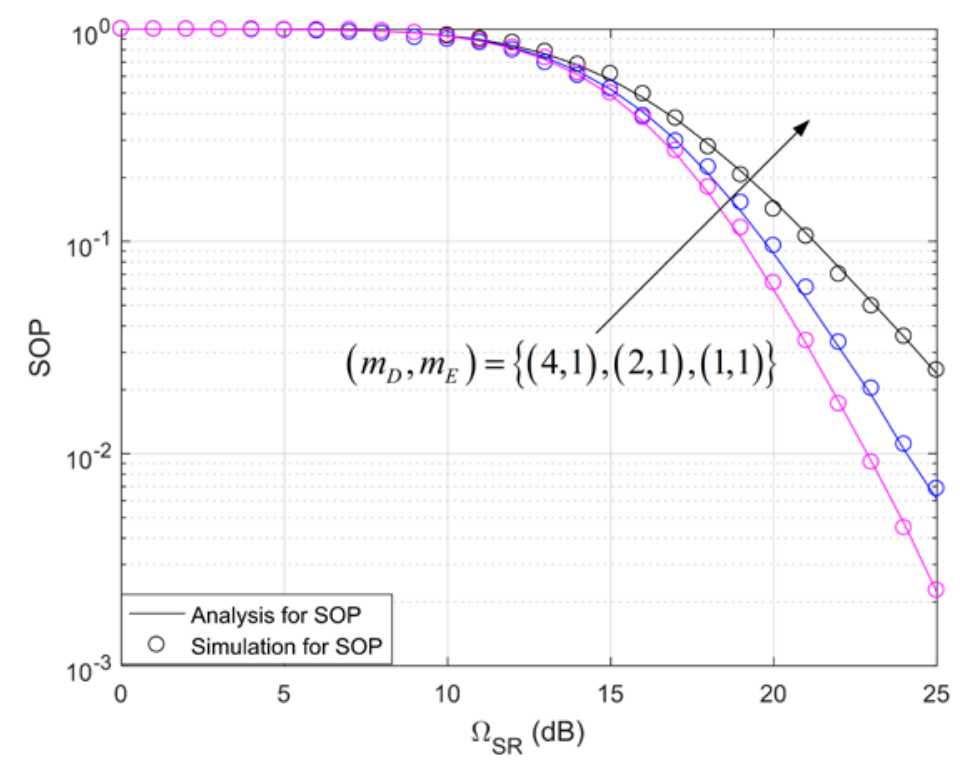

Fig. 6. SOP versus $\Omega_{S R}$ for various values of $\left(m_{D}, m_{E}\right)$ when $k_{D}=k_{E}=2, \mu_{D}=\mu_{E}=2, L=2$. 


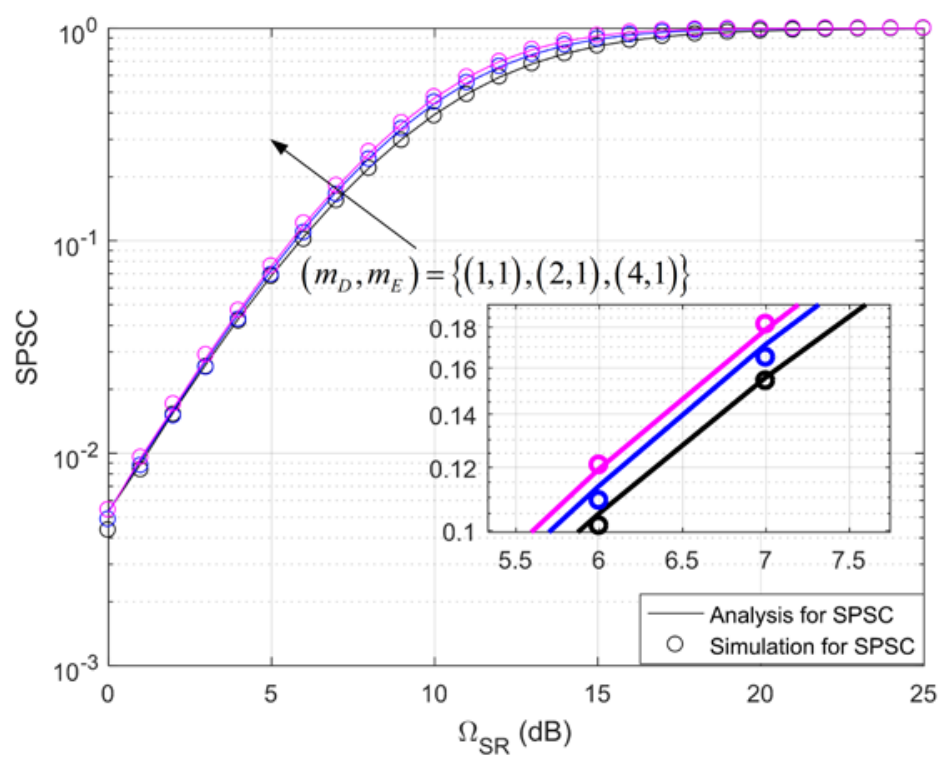

Fig. 7. SPSC versus $\Omega_{S R}$ for various values of $\left(m_{D}, m_{E}\right)$ when $k_{D}=k_{E}=2, \mu_{D}=\mu_{E}=2, L=2$.

Fig. 6 and Fig. 7 describe the SOP and SPSC with changing $\left(m_{D}, m_{E}\right)$ versus $\Omega_{S R}$, in which $m_{i}$ denotes the shaping parameter of Nakagami-m random variable (RV). In Fig. 6, under the condition that $\Omega_{S R}>10 \mathrm{~dB}$ exists, when the value of $m_{D}$ decreases, the value of SOP increases. In Fig. 7, increasing $m_{D}$ will yield increasing SPSC. That is to say, larger $\left(m_{D}, m_{E}\right)$ is beneficial to the improvement of confidentiality on the premise of high SNR regime.

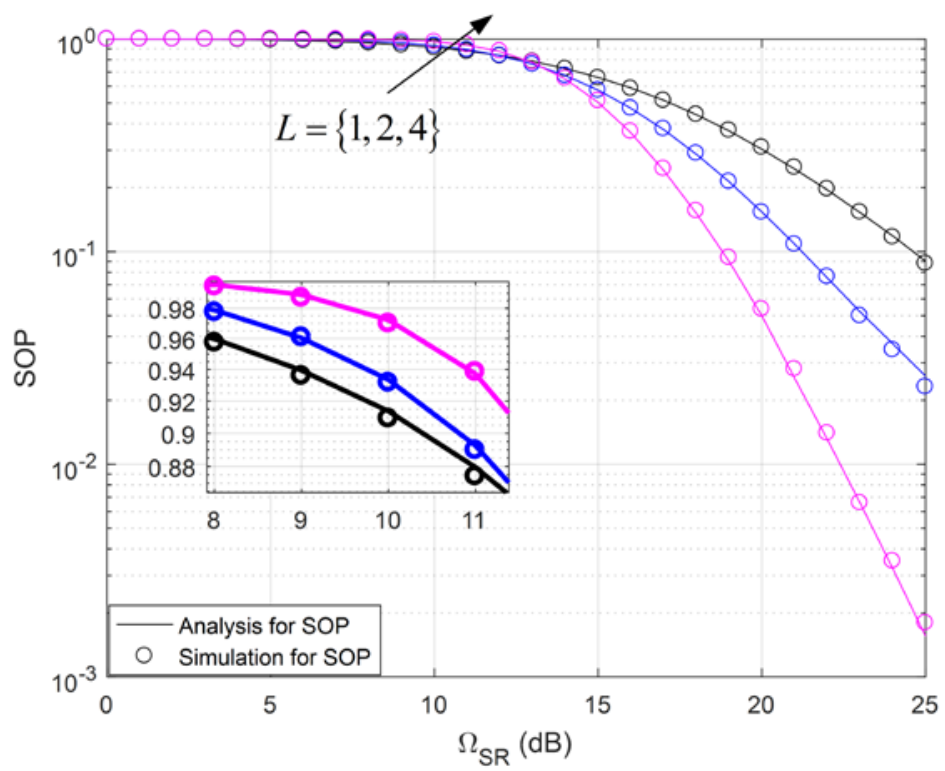

Fig. 8. SOP versus $\Omega_{S R}$ for various values of $L$ when $k_{D}=k_{E}=2, \mu_{D}=\mu_{E}=2, m_{D}=m_{E}=1$. 


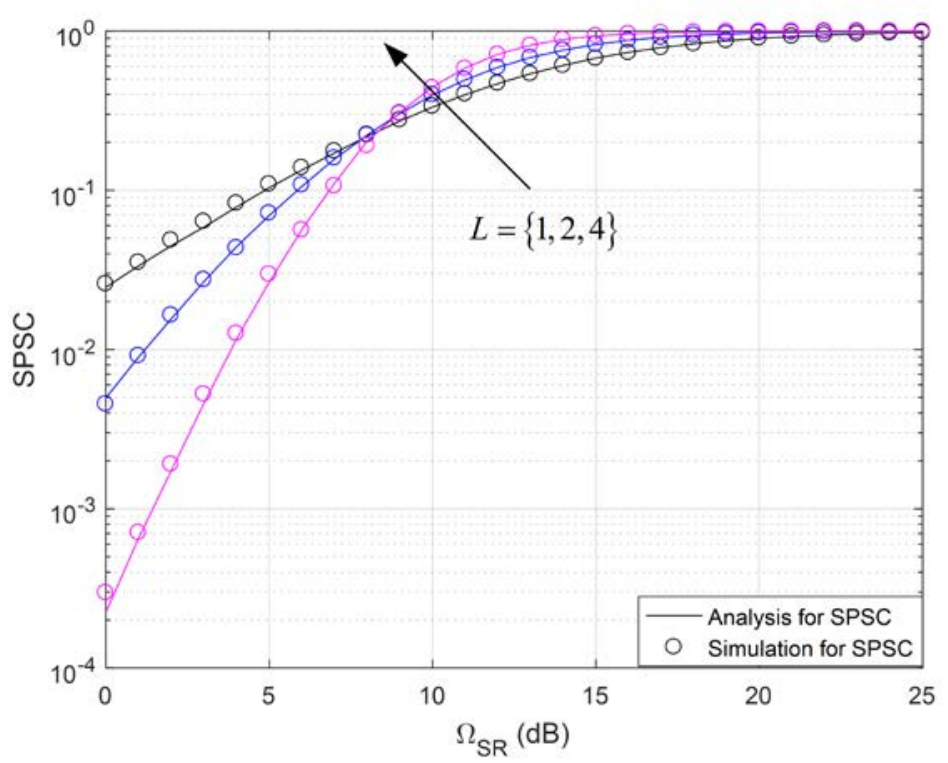

Fig. 9. SPSC versus $\Omega_{S R}$ for various values of $L$ when $k_{D}=k_{E}=2, \mu_{D}=\mu_{E}=2, m_{D}=m_{E}=1$.

As shown in Fig. 8 and Fig. 9, we present the change of SOP and SPSC versus $\Omega_{S R}$ against different values of $L$, From Fig. 8, we can observe that when $\Omega_{S R}>13 \mathrm{~dB}$, the SOP increases with the falling $L$, and vice versa. In Fig. 9, we can see that the value of SPSC increases as increasing $L$ with $\Omega_{S R}>8 \mathrm{~dB}$, and vice versa. The above facts show that in larger SNR regime, the increasing number of antennas is conducive to the improvement of confidentiality in considered relay network, while under smaller SNR regime, the increasing number of antennas causes the decrease of secrecy performance.

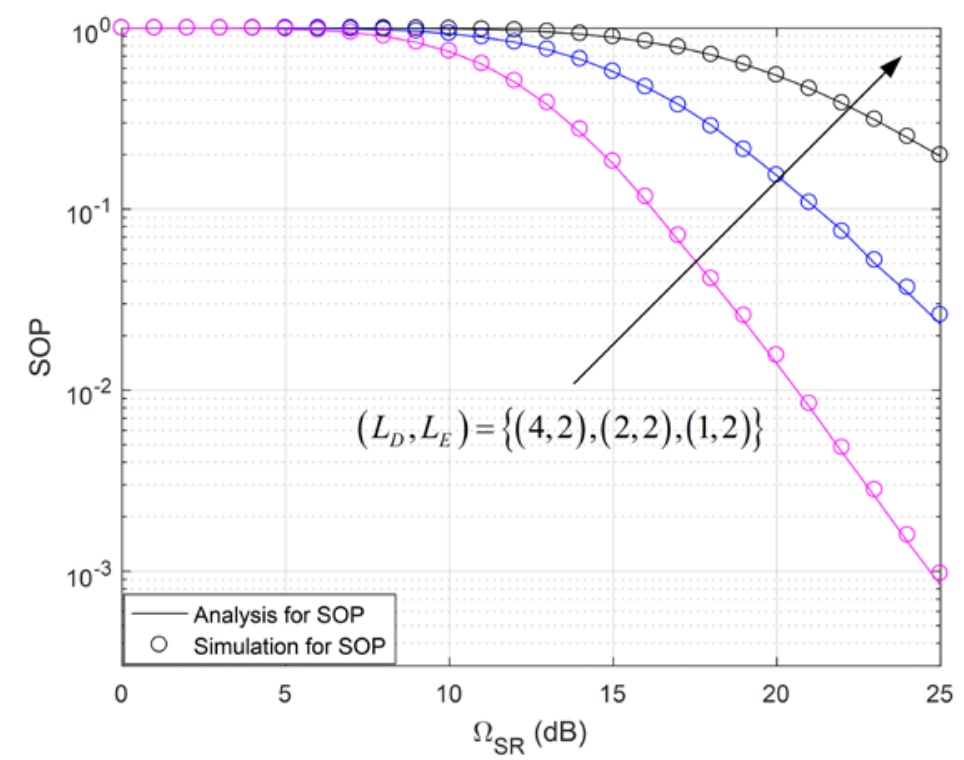

Fig. 10. SPSC versus $\Omega_{S R}$ for various values of $\left(L_{D}, L_{E}\right)$ when $k_{D}=k_{E}=2, \mu_{D}=\mu_{E}=2, m_{D}=m_{E}=1$. 


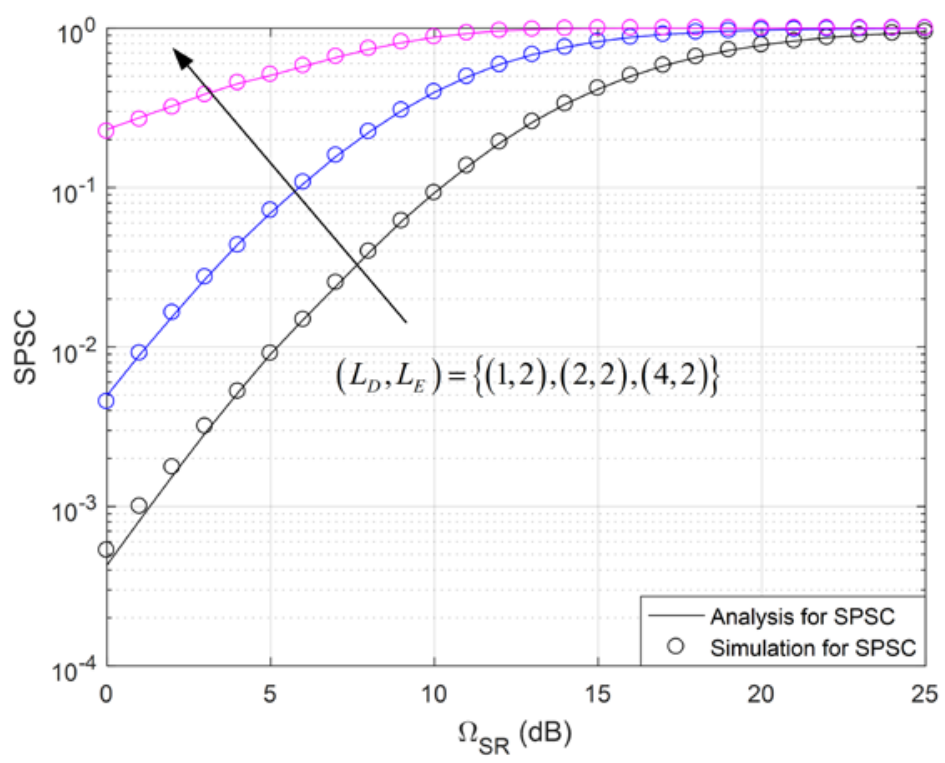

Fig. 11. SPSC versus $\Omega_{S R}$ for various values of $\left(L_{D}, L_{E}\right)$ when $k_{D}=k_{E}=2, \mu_{D}=\mu_{E}=2, m_{D}=m_{E}=1$.

Fig. 10 and Fig. 11 illustrate the changes of SOP and SPSC with $L_{D} \neq L_{E}$, We can find that when the values of other parameters are the same, under $\Omega_{S R}>5 \mathrm{~dB}$, the SOP takes a smaller value with the increase of the ratio of $\left(L_{D}, L_{E}\right)$, while the value of SPSC is larger under arbitrary $\Omega_{S R}$ and larger ratio of $\left(L_{D}, L_{E}\right)$. It can be obtained that when the number of antennas at the legitimate terminal is larger than that at the eavesdropper, the security capability can be significantly improved.

\section{Conclusion}

In this paper, we explore PLS for WCNs over $\kappa-\mu$ shadowed fading channels in term of SOP and SPSC. Based on the proposed SIMO DF relay model, the new theoretical expressions for SOP and SPSC are derived with concise functions. Furthermore, the numerical results are provided to verify the correctness of our theoretical analysis, and the simulation results illustrate that: i) large ratio of $\left(\mu_{D}, \mu_{E}\right)$ and $\left(m_{D}, m_{E}\right)$ can enhance security performance of the considering relay network, while large ratio of $\left(k_{D}, k_{E}\right)$ will reduce the confidentiality of the model. ii) Under the premise of high SNR, large ratio of $\left(L_{D}, L_{E}\right)$ contributes to the improvement of secrecy performance, and vice versa.

\section{References}

[1] A. D. Wyner, “The wire-tap channel,” The Bell System Technical Journal, vol. 54, no. 8, pp. 1355-1387, Oct. 1975. Article (CrossRef Link)

[2] W. Wang, L. Yang, Q. Zhang and T. Jiang, "Securing On-Body IoT Devices By Exploiting Creeping Wave Propagation,” IEEE Journal on Selected Areas in Communications, vol. 36, no. 4, pp. 696-703, Apr. 2018. Article (CrossRef Link) 
[3] W. Wang, S. He, L. Sun, T. Jiang and Q. Zhang, "Cross-Technology Communications for Heterogeneous IoT Devices Through Artificial Doppler Shifts," IEEE Transactions on Wireless Communications, vol. 18, no. 2, pp. 796-806, Feb. 2019. Article (CrossRef Link)

[4] B. Lu, Z. Qin, M. Yang, X. Xia, R. Zhang and L. Wang, "Spoofing Attack Detection Using Physical Layer Information in Cross-Technology Communication," in Proc. of 2018 15th Annual IEEE International Conference on Sensing, Communication, and Networking (SECON), Hong Kong, pp. 1-2, 2018. Article (CrossRef Link)

[5] C. Tang, G. Pan and T. Li, "Secrecy Outage Analysis of Underlay Cognitive Radio Unit Over Nakagami-m Fading Channels," IEEE Wireless Communications Letters, vol. 3, no. 6, pp. 609-612, Dec. 2014. Article (CrossRef Link)

[6] H. Lei, C. Gao, Y. Guo and G. Pan, "On Physical Layer Security Over Generalized Gamma Fading Channels,” IEEE Communications Letters, vol. 19, no. 7, pp. 1257-1260, Jul. 2015. Article (CrossRef Link)

[7] H. Lei, I. S. Ansari, G. Pan, B. Alomair and M. Alouini, "Secrecy Capacity Analysis Over $\alpha-\mu$ Fading Channels,” IEEE Communications Letters, vol. 21, no. 6, pp. 1445-1448, Jun. 2017. Article (CrossRef Link)

[8] X. Pan, H. Ran, G. Pan, Y. Xie and J. Zhang, "On Secrecy Analysis of DF Based Dual Hop Mixed RF-FSO Systems,” IEEE Access, vol. 7, pp. 66725-66730, May. 2019. Article (CrossRef Link)

[9] G. Chen, Y. Gong, P. Xiao and J. A. Chambers, "Physical Layer Network Security in the Full-Duplex Relay System," IEEE Transactions on Information Forensics and Security, vol. 10, no. 3, pp. 574-583, Mar. 2015. Article (CrossRef Link)

[10] K. Guo, K. An, B. Zhang, Y. Huang and D. Guo, "Physical Layer Security for Hybrid Satellite Terrestrial Relay Networks with Joint Relay Selection and User Scheduling,” IEEE Access, vol. 6, pp. 55815-55827, 2018. Article (CrossRef Link)

[11] L. Wu, L. Yang, J. Chen and M. Alouini, "Physical Layer Security for Cooperative Relaying Over Generalized-K Fading Channels," IEEE Wireless Communications Letters, vol. 7, no. 4, pp. 606-609, Aug. 2018. Article (CrossRef Link)

[12] K. P. Peppas, G. C. Alexandropoulos and P. T. Mathiopoulos, "Performance Analysis of Dual-Hop AF Relaying Systems over Mixed $\eta-\mu$ and $\kappa-\mu$ Fading Channels," IEEE Transactions on Vehicular Technology, vol. 62, no. 7, pp. 3149-3163, Sep. 2013. Article (CrossRef Link)

[13] X. Li, J. Li and L. Li, "Performance Analysis of Impaired SWIPT NOMA Relaying Networks Over Imperfect Weibull Channels,” IEEE Systems Journal, vol.14, pp.669-672, 2020. Article (CrossRef Link)

[14] A. Salem, K. A. Hamdi and K. M. Rabie, "Physical Layer Security with RF Energy Harvesting in AF Multi-Antenna Relaying Networks," IEEE Transactions on Communications, vol. 64, no. 7, pp. 3025-3038, Jul. 2016. Article (CrossRef Link)

[15] X. Li, M. Huang, J. Li, Q. Yu, K. Rabie and C. C. Cavalcante, "Secure analysis of multi-antenna cooperative networks with residual transceiver HIs and CEEs," IET Communications, vol. 13, no. 17, pp. 2649-2659, Oct. 2019. Article (CrossRef Link)

[16] X. Li, M. Liu, C. Deng, P. T. Mathiopoulos, Z. Ding and Y. Liu, "Full-Duplex Cooperative NOMA Relaying Systems with I/Q Imbalance and Imperfect SIC," IEEE Wireless Communications Letters. vol. 9, no. 1, pp. 17-20, Jan. 2020. Article (CrossRef Link)

[17] X. Li, J. Li, Y. Liu, Z. Ding and A. Nallanathan, "Residual Transceiver Hardware Impairments on Cooperative NOMA Networks," IEEE Transactions on Wireless Communications, vol. 19, no. 1, pp. 680-695, Jan. 2020. Article (CrossRef Link)

[18] A. Zhao and Z. Ren, "Size Reduction of Self-Isolated MIMO Antenna System for 5G Mobile Phone Applications," IEEE Antennas and Wireless Propagation Letters, vol. 18, no. 1, pp. 152-156, Jan. 2019. Article (CrossRef Link)

[19] W. Zhang and Z. S. He, "Comments on Waveform Optimization for Transmit Beamforming with MIMO Radar Antenna Array,” Transactions on Antennas and Propagation, vol. 66, no. 11, pp. 6463-6463, Nov. 2018. Article (CrossRef Link) 
[20] Y. Yuan, C. Wang, Y. He, M. M. Alwakeel and e. M. Aggoune, “3D Wideband Non-Stationary Geometry-Based Stochastic Models for Non-Isotropic MIMO Vehicle-to-Vehicle Channels,” IEEE Transactions on Wireless Communications, vol. 14, no. 12, pp. 6883-6895, Dec. 2015. Article (CrossRef Link)

[21] L. Zhou and Y. Cao, “The Performance of Physical Layer Security over K Fading SIMO Channel," in Proc. of 2018 IEEE 18th International Conference on Communication Technology (ICCT), Chongqing, 2018, pp. 145-149, 2018. Article (CrossRef Link)

[22] A. H. A. El-Malek, A. M. Salhab, S. A. Zummo and M. Alouini, "Enhancing Physical Layer Security of Multiuser SIMO Mixed RF/FSO Relay Networks with Multi-Eavesdroppers,” in Proc. of 2016 IEEE Globecom Workshops (GC Wkshps), Washington, DC, pp. 1-7, 2016. Article (CrossRef Link)

[23] G. Pan, C. Tang, T. Li and Y. Chen, "Secrecy Performance Analysis for SIMO Simultaneous Wireless Information and Power Transfer Systems,” IEEE Transactions on Communications, vol. 63, no. 9, pp. 3423-3433, Sep. 2015. Article (CrossRef Link)

[24] H. Lei, C. Gao, I. S. Ansari, Y. Guo, G. Pan and K. A. Qaraqe, “On Physical-Layer Security Over SIMO Generalized-K Fading Channels,” IEEE Transactions on Vehicular Technology, vol. 65, no. 9, pp. 7780-7785, Sep. 2016. Article (CrossRef Link)

[25] H. Wang, L. Xu, W. Lin, P. Xiao and R. Wen, "Physical Layer Security Performance of Wireless Mobile Sensor Networks in Smart City,” IEEE Access, vol. 7, pp. 15436-15443, 2019. Article (CrossRef Link)

[26] L. Kong, G. Kaddoum and Z. Rezki, "Highly Accurate and Asymptotic Analysis on the SOP Over SIMO $\alpha-\mu$ Fading Channels," IEEE Communications Letters, vol. 22, no. 10, pp. 2088-2091, Oct. 2018. Article (CrossRef Link)

[27] L. Moreno-Pozas, F. J. Lopez-Martinez, S. L. Cotton, J. F. Paris and E. Martos-Naya, “Comments on Human Body Shadowing in Cellular Device-to-Device Communications: Channel Modeling Using the Shadowed $\kappa-\mu$ Fading Model,” IEEE Journal on Selected Areas in Communications, vol. 35, no. 2, pp. 517-520, Feb. 2017. Article (CrossRef Link)

[28] J. Zhang, X. Li, I. S. Ansari, Y. Liu and K. A. Qaraqe, "Performance Analysis of Dual-Hop DF Satellite Relaying over $\kappa-\mu$ Shadowed Fading Channels," in Proc. of IEEE Wireless Communications and Networking Conference (WCNC), San Francisco, CA, pp. 1-6, 2017. Article (CrossRef Link)

[29] Y. J. Chun, S. L. Cotton, H. S. Dhillon, F. J. Lopez-Martinez, J. F. Paris and S. K. Yoo, “A Comprehensive Analysis of 5G Heterogeneous Cellular Systems Operating over $\kappa-\mu$ Shadowed Fading Channels," IEEE Transactions on Wireless Communications, vol. 16, no. 11, pp. 6995-7010, Nov. 2017. Article (CrossRef Link)

[30] J. F. Paris, "Statistical characterization of $\kappa-\mu$ shadowed fading," IEEE Transactions on Vehicular Technology, vol. 63, no. 2, pp. 518-526, Feb. 2014. Article (CrossRef Link)

[31] D. Sadhwani, "Simple and Tightly Approximated Integrals Over $\kappa-\mu$ Shadowed Fading Channel with Applications," IEEE Transactions on Vehicular Technology, vol. 67, no. 10, pp. 10092-10096, Oct. 2018. Article (CrossRef Link)

[32] R. H. Shaik and R. Naidu K, "Performance Analysis of Multi-hop Cooperative System under $\kappa-\mu$ Shadowed Fading Channels," in Proc. of 2019 International Conference on Communication and Signal Processing (ICCSP), Chennai, India, pp. 0587-0591, 2019. Article (CrossRef Link)

[33] M. S. Aloqlah, I. E. Atawi and M. F. Al-Mistarihi, "On the Performance of Fixed-gain Amplify-and-forward Dual-hop Relay Systems with Beamforming Under $\kappa-\mu$ Shadowed Fading," in Proc. of 2015 IEEE 26th Annual International Symposium on Personal, Indoor, and Mobile Radio Communications (PIMRC), Hong Kong, pp. 814-818, 2015. Article (CrossRef Link)

[34] M. Srinivasan and S. Kalyani, "Secrecy Capacity of $\kappa-\mu$ Shadowed Fading Channels," IEEE Communications Letters, vol. 22, no. 8, pp. 1728-1731, Aug. 2018. Article (CrossRef Link)

[35] S. Kumar and S. Chouhan, "Outage probability analysis of cognitive decode-and-forward relay networks over $\kappa-\mu$ Shadowed Channels,” in Proc. of 2015 21st Asia-Pacific Conference on Communications (APCC), Kyoto, pp. 433-437, 2015. Article (CrossRef Link) 
[36] A. Hussain, S. Kim and S. Chang, "Dual-Hop Variable-Gain AF Relaying with Beamforming over $\kappa-\mu$ Shadowed Fading Channels," in Proc. of 2016 IEEE Global Communications Conference (GLOBECOM), Washington, DC, pp. 1-6, 2016. Article (CrossRef Link)

[37] J. Sun, X. Li, M. Huang, Y. Ding, J. Jin and G. Pan, "Performance analysis of physical layer security over $\kappa-\mu$ shadowed fading channels,” IET Communications, vol. 12, no. 8, pp. 970-975, 2018. Article (CrossRef Link)

[38] J. Sun, H. Bie, X. Li, J. Zhang, G. Pan and K. M. Rabie, "Secrecy Performance Analysis of SIMO Systems Over Correlated $\kappa-\mu$ Shadowed Fading Channels,” IEEE Access, vol. 7, pp. 86090-86101, 2019. Article (CrossRef Link)

[39] A. Hussain, S. Kim and S. Chang, "Dual-Hop Variable-Gain AF Relaying with Beamforming over $\kappa-\mu$ Shadowed Fading Channels," in Proc. of 2016 IEEE Global Communications Conference (GLOBECOM), Washington, DC, pp. 1-6, 2016. Article (CrossRef Link)

[40] M. Poreddy, T. M. C. Chu and H. Zepernick, "On Outage Probability of Cooperative Cognitive Radio Networks over $\kappa-\mu$ Shadowed Fading,” in Proc. of 2017 IEEE Wireless Communications and Networking Conference (WCNC), San Francisco, CA, pp. 1-6, 2017. Article (CrossRef Link)

[41] P. N. Son and H. Y. Kong, "Cooperative communication with energy-harvesting relays under physical layer security,” IET Communications, vol. 9, no. 17, pp. 2131-2139, Nov. 2015. Article (CrossRef Link)

[42] Y. Chen, "Energy-Harvesting AF Relaying in the Presence of Interference and Nakagami- $m$ Fading," IEEE Transactions on Wireless Communications, vol. 15, no. 2, pp. 1008-1017, Feb. 2016. Article (CrossRef Link)

[43] Alan Jeffrey and Daniel Zwillinger, Table of Integrals, Series, and Products, 7th ed., Academic Press, San Diego, USA, 2007. Article (CrossRef Link)

[44] J. Zhang, X. Chen, K. P. Peppas, X. Li and Y. Liu, "On High-Order Capacity Statistics of Spectrum Aggregation Systems Over $\kappa-\mu$ and $\kappa-\mu$ Shadowed Fading Channels," IEEE Transactions on Communications, vol. 65, no. 2, pp. 935-944, Feb. 2017. Article (CrossRef Link)

[45] M. Bloch, J. Barros, M. R. Rodrigues and S. W. McLaughlin, "Wireless information-theoretic security,” IEEE Transactions on Information Theory, vol. 54, no. 6, pp. 2515-2534, Jun. 2008. Article (CrossRef Link) 


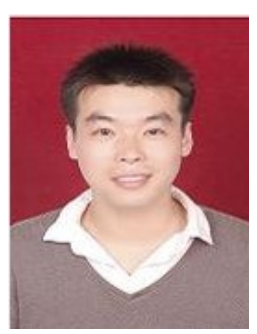

Jiangfeng Sun received the M.S. degree in communication and information system from Zhengzhou University in 2009. He is currently pursuing the Ph.D. degree in information and communication engineering with the Beijing University of Posts and Telecommunications, Beijing, China. His current research interests include physical layer security, cooperative communications and the performance analysis of fading channels.

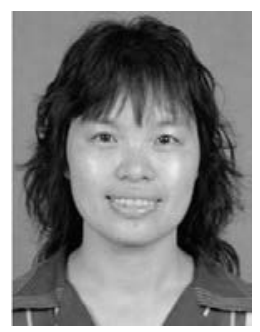

Hongxia Bie received the Ph.D. degree from Jilin University, China, in 2000. She is currently a Professor with the School of Information and Communication Engineering, Beijing University of Posts and Telecommunications. Her main research interests include physical layer security, cooperative communications, multimedia information processing, and wireless data transmission.

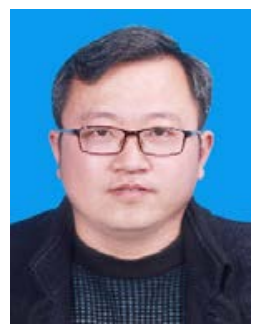

Xingwang Li (Senior Member, IEEE) received the B.Sc. degree from Henan Polytechnic University, in 2007, the M.Sc. degree from the University of Electronic Science and Technology of China, in 2010, and the Ph.D. degree from the Beijing University of Posts and Telecommunications in 2015. From 2010 to 2012, he was working with Comba Telecom Ltd., Guangzhou, China, as an Engineer. From 2017 to 2018, he was a Visiting Scholar with Queen's University Belfast, Belfast, U.K. He is currently an Associate Professor with the School of Physics and Electronic Information Engineering, Henan Polytechnic University, Jiaozuo, China. His research interests include MIMO communication, cooperative communication, hardware constrained communication, non-orthogonal multiple access, physical layer security, unmanned aerial vehicles, and the Internet-of-Things. He is currently an Editor on the Editorial Board of IEEE ACCESS, Computer Communications, Physical Communication, and KSII Transactions on Internet and Information Systems. He is also a Lead Guest Editor of the special issue on Recent Advances in Physical Layer Technologies for $5 \mathrm{G}$ Enabled Internet of Things of Wireless Communications and Mobile Computing. He has served as many TPC/Co-Chair, such as IEEE GLOBECOM, IEEE/CIC ICCC, IEEE WCNC, IEEE VTC, and IEEE/IET CSNDSP. 